\title{
Soft Robotic Surrogate Lung
}

\author{
Olivier Ranunkel and Firat Güder*
}

Department of Bioengineering, Imperial College London, London SW7 2AZ, United Kingdom

\author{
Hari Arora*(0) \\ College of Engineering, Swansea University, Swansea SA1 8EN, United Kingdom
}

\section{Supporting Information}

ABSTRACT: Previous artificial lung surrogates used hydrogels or balloon-like inflatable structures without reproducing the alveolar network or breathing action within the lung. A physiologically accurate, air-filled lung model inspired by soft robotics is presented. The model, soft robotic surrogate lung (SRSL), is composed of clusters of artificial alveoli made of platinum-cured silicone, with internal pathways for air flow. Mechanical tests in conjunction with full-field image and volume correlation techniques characterize the SRSL behavior. SRSLs enable both healthy and pathological lungs to be studied in idealized cases. Although simple in construction, the connected airways demonstrate clearly the importance of an inflatable network for capturing basic lung behavior (compared to more simplified lung surrogates). The SRSL highlights the potentially damaging nature of local defects caused by occlusion or overdistension (present in conditions such as chronic obstructive pulmonary disease). The SRSL is developed as a potential upgrade to conventional surrogates used for injury risk predictions in trauma. The deformation of the SRSL is evaluated against blast trauma using a shock tube. The SRSL was compared to other conventional trauma surrogate materials and showed greatest similarity to lung tissue. The SRSL has the potential to complement conventional biomechanical studies and reduce animal use in basic biomechanics studies, where high severity protocols are used.

KEYWORDS: lung, biomechanics, soft robotics, trauma, image correlation

\section{INTRODUCTION}

Size, organization, and the multiphase nature make the lung a complex organ. The human lung is composed of millions of alveolar ducts, each of which terminates in the acinus, a grouping of elastic sacs; these elastic sacs, called alveoli, are the basic unit of ventilation. The adult human lung can contain over 300 million alveoli. ${ }^{1,2}$ Lungs, while being elastic and protected within the body, remain a vulnerable organ that can become damaged through disease or trauma, resulting in reduced functional capacity. Alveolar walls within lungs with augmented structure, material behavior, and reduced functional capacity can lead to unusual stresses and strains ${ }^{3}$ forming during respiration that can, in fact, induce or exacerbate respiratory pathologies. Yet, currently, our ability to model the mechanical behavior of this network within the lung is limited. ${ }^{4}$

Away from pure physiological studies, in the realms of bulk tissue biomechanics and protective armor development against traumatic injury, various materials are being investigated as synthetic lung surrogates. These surrogates aim to mimic the basic mechanical properties of lungs in terms of, for example, stiffness and density. There is an interest in using hydrogels because of their adaptability, high compliance, and comparable bulk mechanical characteristics. ${ }^{5}$ Similarly, polyurethane foam with fluid-filled gelatin microcapsules have also been

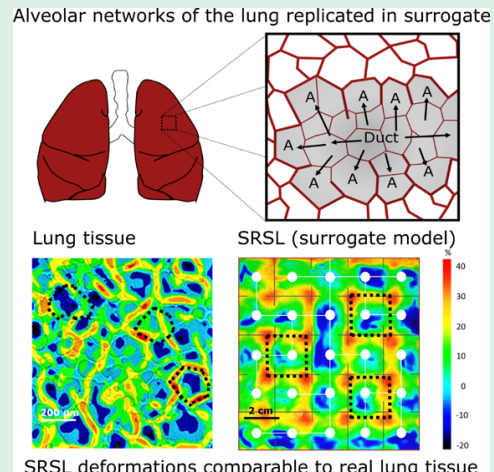

SRSL deformations comparable to real lung tissue investigated because it mimics the dynamic response of the lung. ${ }^{6}$ Hydrogels and foams, however, do not capture the connectivity and interdependency of the alveolar network present in the lungs. Alveoli form a structured connected network rather than isolated or randomized pores (Figure 1). On a microscopic scale, current hydrogel surrogates used in trauma (cast ballistic gelatin) do not capture the microstructure of lungs. At this scale, the only similarity between the microstructure of hydrogels and alveoli is pore size. The rise of additive manufacturing methods has opened new possibilities regarding the manufacture process. ${ }^{7}$ For example, the resolution of prints has approached the $10 \mathrm{~s}$ of micron scale in recent times in tough and flexible hydrogels. ${ }^{8,9}$ However, scaling-up the manufacturing process for large structures with such fine details will be the next challenge.

Functional aspects of lung using flexible membranes have progressed through development of artificial gas exchange membranes and lung-on-a-chip. ${ }^{10}$ Such models capture function, enabling detailed study of mass transfer across alveolar wall segments within cell culture models. The three-

Received: November 26, 2018

Accepted: March 8, 2019

Published: March 8, 2019 


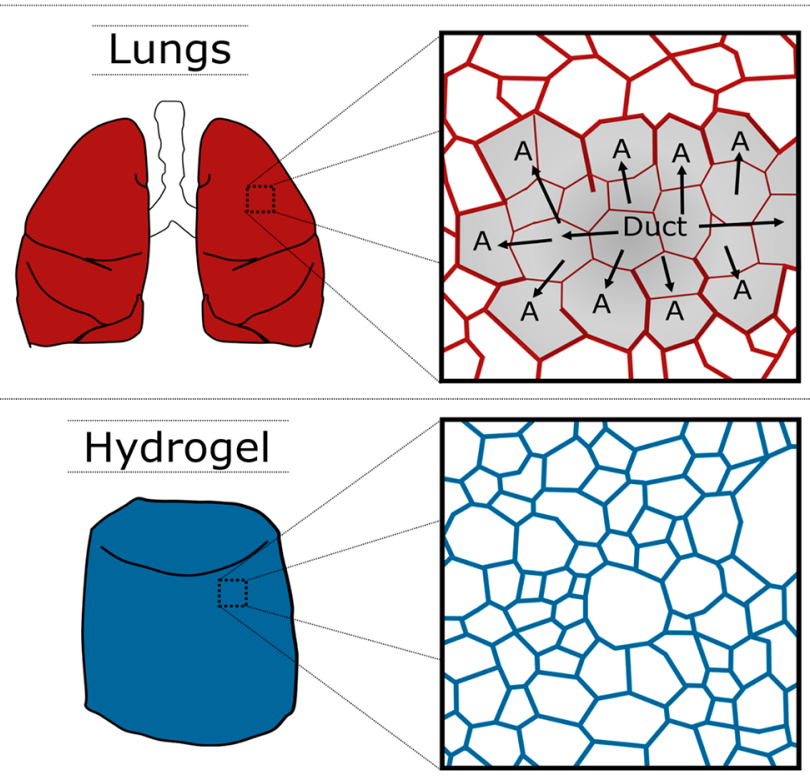

Figure 1. Schematic comparison of lung and hydrogel microstructures. While the lung tissue contains airways connecting alveoli together, hydrogels only contain isolated pores. ${ }^{3}$

dimensional biomechanical representation of the air-spaces and their interaction is of focus here. Soft robotic models are now being developed for biomechanical applications. Organs such as the heart ${ }^{11-13}$ have exploited soft robotics in differing guises to good effect. Soft robotics lends itself ideally toward lung biomimicry due to the pneumatic nature of the devices. In trauma simulators, where hydrogels are typically used, there is an absence of structured pathways for air to move. Hydrogels can be produced with porosity and random interconnections between pores resulting in open-celled foam structures, ${ }^{14}$ closed-cell foam structures, or porous solids. ${ }^{6}$ Randomized pathways and discrete air-spaces, therefore, cannot simulate the same dynamic mechanical behavior of a real lung. Alternative approaches are required to explicitly capture the airway dynamics present within the hierarchical tree-like structure of real lungs.

While previous models have treated the lung as a piece of porous material, lungs are soft actuators and contain a compliant and complex network of airways, which inflate and deflate through the movement of the diaphragm and thoracic cage. Inspired by animals such as the octopus or starfish, soft robotics use soft and flexible materials to create pneumatically or hydraulically controlled actuators that can perform complex tasks that conventional "hard" robots cannot. ${ }^{15,16}$ Some of the applications of soft robots in biomedical sciences include implants, devices for rehabilitation, and assistive exoskeletons to improve the weight-bearing capacity in humans. ${ }^{17,18}$ Soft robots are mainly constructed using highly deformable materials such as silicone or urethane elastomers. Although silicone or urethane elastomers and their variations match the elastic and rheological properties of biological tissue, ${ }^{19,20}$ there have not been any soft robots modeling lung behavior.

The development of a soft robotic surrogate lung (SRSL) is reported here. Specifically, clusters of artificial alveoli (AA), replicating the dynamic movement of alveoli, are presented. The SRSL is validated against real lung tissue mechanical data. The use of soft robots made of silicones enables fabrication of active lung surrogates. They will capture the interaction of the air and the network of soft solid alveolar walls. In comparison to passive designs, ${ }^{5,7,21,22}$ AA (Figure 2) can accurately mimic the mechanical properties and the dynamic behavior of lungs, with true biomechanical-fidelity.

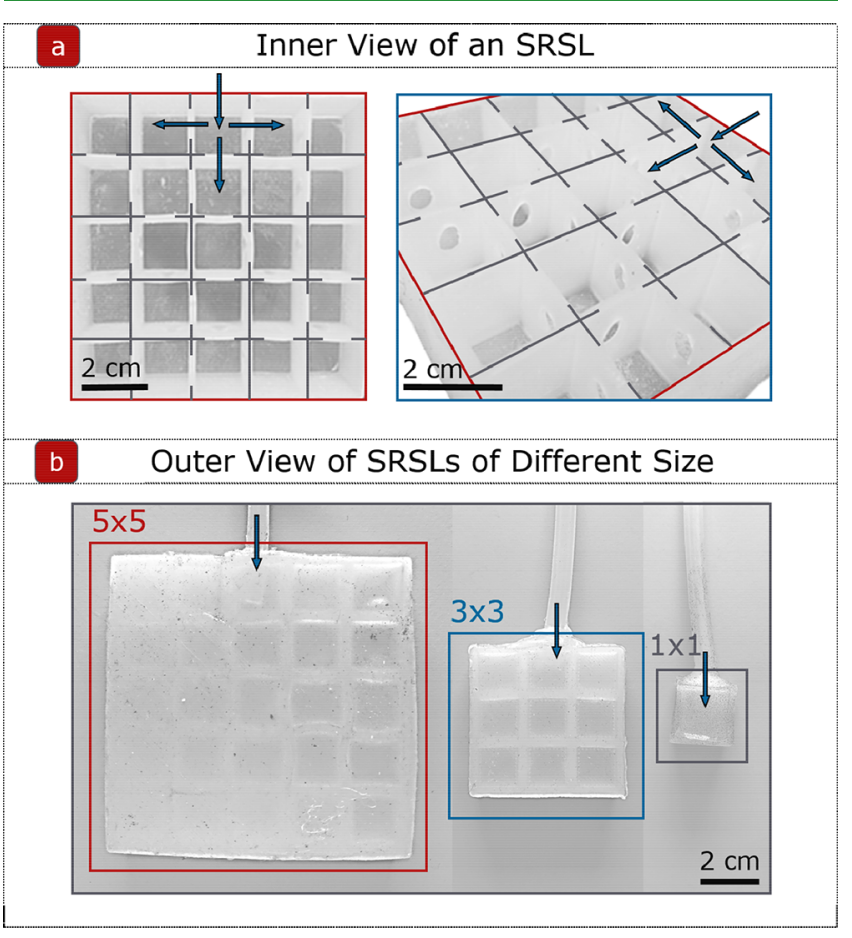

Figure 2. (a) Top and isometric views of the internal structure of a 5 $\times 5$ SRSL with a network of pathways. The arrows indicate the pathway of air into the model. The pathways are created by the holes in the walls visible on the right-hand picture. (b) Three SRSLs containing a different number of AA.

\section{EXPERIMENTAL SECTION}

2.1. Material Selection. Lung tissue can strain up to $400 \%$ before failure in excised animal samples ${ }^{23}$ and up to $\sim 80 \%$ during a respiratory cycle. ${ }^{24}$ Because of their similarity to the mechanical properties of mammalian tissue and ease-of-fabrication, silicone materials were selected for the construction of the SRSL. ${ }^{25}$ Platinum-cured silicones were used (e.g., Ecoflex 00-10), sourced from Smooth-On. In the literature, other research groups have also used this class of materials due to its high compliance and elongation in physiological applications. ${ }^{26,27}$

2.2. Tensile Testing of Silicone Elastomers. Test samples were prepared according to ASTM D454 for Ecoflex 00-10, Ecoflex 0030, porous Ecoflex $00-30$ with $40 \%$ porosity, Figure S-1, Dragon Skin 30 and Dragon Skin 50. Samples were tested under tension using a $1 \mathrm{kN}$ Instron testing machine.

2.3. Alveolar Geometry and Structural Organization. The alveolar geometry of the lungs has evolved to create a high packing density of alveoli. The lung structure works to maximize the number of alveoli and surface area, while minimizing the average length of a pathway. The design of the SRSL (Figure 2) accounted for the rules for the organization and connection between each AA observed in nature. ${ }^{28}$ According to these rules, (i) an AA can only connect to its three nearest neighboring AA; (ii) an AA must be connected by one single possible pathway to the entrance; and (iii) the path from the inlet to any node must not have bends greater than $90^{\circ}$. Alveoli have been generally modeled as truncated octahedra that produce enhanced stiffness along the edges of the structure. ${ }^{28}$ The regularsized cuboidal arrangement chosen here is simplified in geometry and 
will exhibit a higher degree of anisotropy due to the fewer number of faces/edges.

2.4. Manufacture of the SRSL. Similar to the fabrication of many soft actuators, 3D-printed polylactic acid (PLA) molds (Figure 3a)

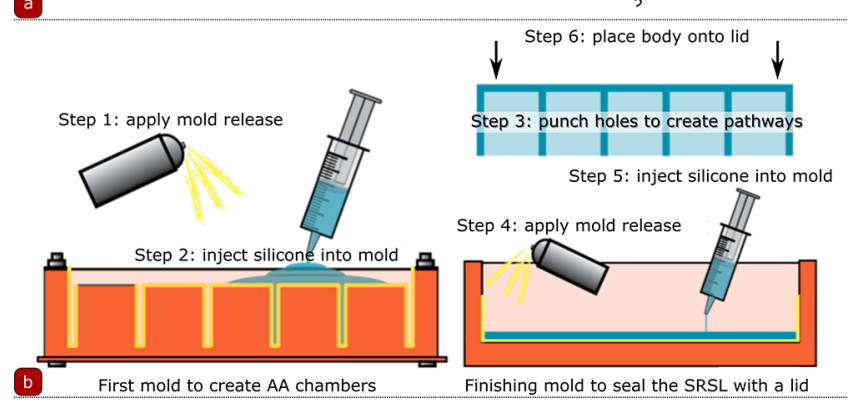

Figure 3. SRSL Manufacture: (a) computer aided design rendering of the 3D printed PLA mold for a $5 \times 5$ SRSL; (b) step-by-step illustration of SRSL manufacture and assembly process.

were designed for producing the SRSL via a casting method. ${ }^{29}$ 3D printing molds provide the possibility to custom shape the SRSL. Any rigid printing material can be used, and PLA was used here on Ultimaker 2 printers. The mold exists in two parts for ease of removal of the cured silicone part. Molds were clamped for a repeatable seal using nuts and bolts around the perimeter of the mold (Figure 3a).

The mold was sprayed twice with a layer of mold release (Ambersil lubricant aerosol) at $10 \mathrm{~min}$ intervals to allow the mold to dry to help the silicone release once cured. The platinum-cured silicones used came in two parts: A and B. Both parts were mixed into a syringe with equal volume. The silicone (Ecoflex 00-30) was poured into the mold, which was in turn placed into a vacuum chamber for $30 \mathrm{~min}$ to remove any air bubbles trapped in the silicone. Later, the mold was placed into the oven at $55^{\circ} \mathrm{C}$ (glass transition temperature of PLA is $65{ }^{\circ} \mathrm{C}$ ) for $90 \mathrm{~min}$ opened, and the part removed. Holes were punched in selected walls of the cured part to create the pathways, visible in Figure 2a. The second mold was prepared in a similar manner to close the SRSL (Figure 3b). The SRSL is then pierced with a needle to create the inlet, where a silicone tube of $3 \mathrm{~mm}$ outer diameter and $1 \mathrm{~mm}$ inner diameter was placed. To ensure an airtight seal, fast curing silicone (Polycraft T-20 Translucent Silicone Rubber from MBFG) was used to bond the tubing to the SRSL.

2.5. Pressure-Volume Measurements of SRSL. Pressurevolume (PV) measurements are a mechanical diagnostic tool to assess the ventilation behavior, that is, stiffness/compliance of lungs. ${ }^{30} \mathrm{~A}$ custom-built, controlled inflation tester $(\mathrm{CiT})^{31}$ (Figure S-2) was used to deliver a controlled volume of air and monitor internal pressure via a pressure transducer (Omegadyne PX309). The compliance of the air in the ventilation system (i.e., all air not in the test sample) was corrected for to enable the capture of true PV characteristics of the test samples (lungs and SRSL). The experimental protocol was controlled using LabVIEW.

2.6. SRSL Strain Measurements. Full-field strain plots of the SRSL surface were obtained for the duration of each test by employing 3D digital image correlation (DIC) methods. Two cameras were positioned in stereo, facing the speckled test sample (black speckles on the matte white background of the natural silicone color). The speckle pattern was sized according to the resolution of cameras (5 MP) and was evenly distributed over the test sample, but randomly distributed within the frames of the facets for reliable image correlation. Cameras were calibrated, using a known pattern, to allow the recorded test images to be processed in ARAMIS (GOM $\mathrm{GmbH})$, the DIC software used to perform the image correlation. Cameras sampled at $1 \mathrm{fps}$ at full resolution $(2448 \times 2050$ pixels $)$. Given the quasi-static nature of the experiment, it was possible to operate at full resolution (keeping spatial resolution high, i.e., detail of analysis), while capturing the images at a suitable temporal resolution. As real lungs are contained within a rib cage and, specifically, alveoli are constrained by their neighbors, a near infinitely rigid boundary was applied to the SRSL edges during all tests (a 3D printed square frame).

2.7. Measurement of Strains in Rat Lung Tissue. The synchrotron X-ray microtomography experiments were conducted at the Diamond-Manchester Imaging Branchline I13-2 of Diamond Light Source (DLS), UK, on female cadaveric Sprague-Dawley rats $(200-280 \mathrm{~g}){ }^{31}$ No live animals or human subjects were tested in this study. Cadaveric tissue was obtained from animals from a registered animal supplier. Procedures were approved by the Imperial College London ethical review process and strictly conformed to the Animals (Scientific Procedures) Act 1986 UK Home Office guidelines, which also fulfill the US NIH Guide for the Care and Use of Laboratory Animals. Two volumetric images were acquired at two different volumes for a healthy excised lung sample. Low lung volume was studied to enable similar strain magnitudes to be achieved in the SRSL using the same ventilator system. Two images are required to compute a single strain field as the correlation algorithm tracks the changes between the reference and the deformed image. Volumetric strains were computed using digital volume correlation (DVC) methods (DaVis StrainMaster by LaVision). A cross-section of inplane strains produced using the DVC (i.e., extracting a $2 \mathrm{D}$ slice of data from the 3D data set) was compared to the DIC images acquired on the surface of the SRSL.

2.8. Study of Blast Injuries. A shock tube was used in the Centre of Blast Injury Studies (CBIS) to replicate the forces exerted during a blast on biological tissue and surrogates. ${ }^{31-33}$ This shock tube was formed of sections of stainless steel (3.8 m total length), with an internal diameter of $59 \pm 1 \mathrm{~mm}$. Compressed air was used to pressurize the driver section. A single-diaphragm configuration was used. Diaphragm material was a polymer film (Mylar) of 50 and 100 $\mu \mathrm{m}$ thickness. All biological and nonbiological samples (silicones, $10 \%$ gelatin and an excised rat lung embedded in $10 \%$ gelatin to couple the pressure signal) were subjected to shockwaves with a peak overpressure of $120 \mathrm{kPa}$. A Phantom v611 high-speed camera was used to visualize the shockwave impinging on the front face of the samples at $28000 \mathrm{fps}$ (with a resolution of $512 \times 384$ pixels). A pressure transducer (Dytran $2300 \mathrm{~V} 4$ ) was mounted behind the tested samples to record the transmitted pressure.

\section{RESULTS AND DISCUSSION}

3.1. Selection of Silicone Elastomers for Fabrication of SRSL. To create an accurate surrogate model of the lungs, the mechanical properties must be considered at two scales: (i) the mechanical properties of the alveolar walls (the solid); and (ii) the mechanical properties of the complete lung model, that is, the alveolar walls and air together (the foam). A material with mechanical properties similar to the alveolar walls was needed to construct an SRSL.

To find the most suitable material that matches the mechanical properties of the alveolar walls, the stress-strain behavior of several silicone variants was tested under tension: Ecoflex 00-10, Ecoflex 00-30, Dragon Skin 30, and Dragon Skin 50. These materials were compared against elastin and collagen, the primary structural elements present in the lung (Figure 4a). While Ecoflex 00-10 exhibited mechanical characteristics similar to elastin, Dragon Skin 50 behaved like collagen. ${ }^{34}$ Mammalian lungs have a collagen-to-elastin ratio of 


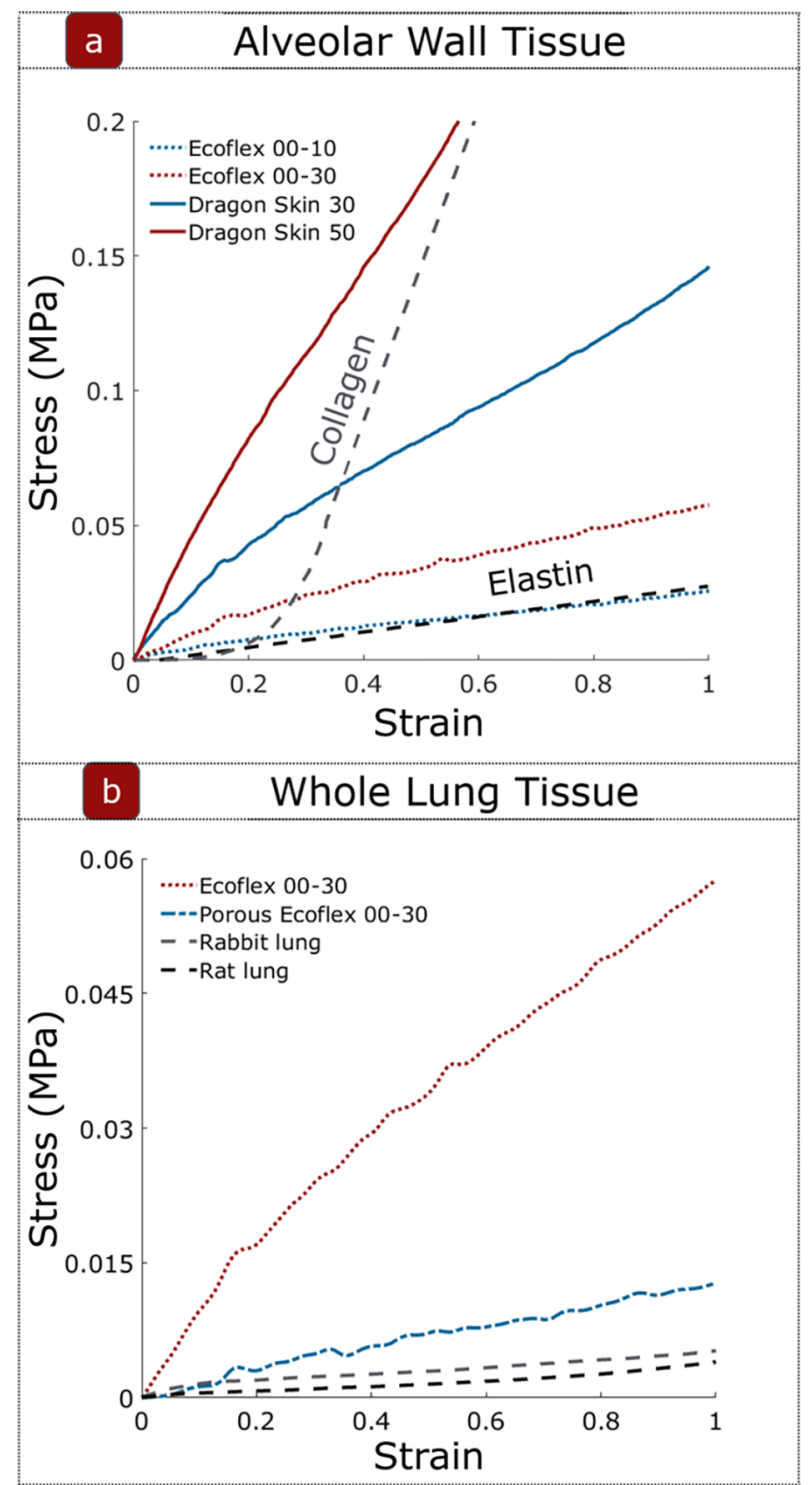

Figure 4. (a) Stress-strain comparison of various silicones with respect to elastin and collagen. ${ }^{35}$ (b) Comparison of the stress-strain behavior of nonporous and porous silicones with animal whole lung biopsies. $^{23}$

approximately $2: 1 ;^{35}$ therefore, using a law of mixtures approach, Ecoflex 00-30 was selected as it falls in between Ecoflex 00-10 and Dragon Skin 50 with a bias toward the stiffness of elastin (Ecoflex 00-10). The specific ratio of collagen to elastin varies in nature and throughout a given lung itself. Here, the SRSL model was manufactured using one material and a (near) fixed thickness throughout. The model had, therefore, a fixed set of structural properties. Future iterations, however, may explore spatial variation of mechanical properties, for example, creating multimaterial systems.

Given the SRSL will be an air-filled structure, one final check to ensure the material selected was fit for purpose was to assess its foam properties. Bulk mechanical tests of lung tissue often involve taking biopsies of lung tissue for testing. Such samples will test the solid tissue but also to some extent the air-filled behavior of the porous architecture. Therefore, to assess the suitability of Ecoflex 00-30 to being used to model lung tissue (Figure $4 b$ ), a set of preliminary tests were performed on porous Ecoflex 00-30. The tensile modulus of solid (negligible porosity) Ecoflex 00-30, was more than one order of magnitude higher than animal lung tissue. This is expected since the wall material of our SRSL (solid) will be stiffer than the final full SRSL model (the foam-like structure). Considering foam theory, ${ }^{36}$ the solid modulus is always going to be higher than that of the foam equivalent. To gauge the expected bulk material performance of the air-filled SRSL structure, a set of Ecoflex 00-30 samples with $40 \%$ porosity was produced. By mixing the silicone polymer with crystalline $\mathrm{CuSO}_{4}$ salt and dissolving the salt in distilled water at $90{ }^{\circ} \mathrm{C}$, porous samples were produced ${ }^{37}$ (Figure S-1). The tensile modulus of porous silicone compared better with the tensile modulus of animal lung tissue; ${ }^{23}$ however, the pores within the material were not structured, continuously interconnected, controlled, and could not be actuated. In any case, the system proposed is versatile and offers multiple degrees of freedom to produce new variants of the current robotic surrogate. Furthermore, at present, there is no industrial grade Ecoflex with a specified porosity available commercially. Solid Ecoflex 00-30, therefore, was used as the wall material of the SRSL for the remainder of the study to create a material with controlled pore geometry and pathway architecture, which can also be actuated pneumatically.

3.2. Determining Size and Pathway Geometry of SRSL. To select the correct geometry to build the SRSL, pressure-volume (PV) measurements were performed. PV measurements are a diagnostic tool to characterize the mechanical ventilation behavior of lungs. ${ }^{30}$ SRSLs consisted of $1 \times 1 \mathrm{AA}, 3 \times 3 \mathrm{AA}$, and $5 \times 5 \mathrm{AA}$; each AA measured $18 \times$ $18 \times 18 \mathrm{~mm}^{3}$ with wall thickness $2 \mathrm{~mm}$ (Figure $2 \mathrm{~b}$ ). The SRSLs are scaled-up by three orders of magnitude in size compared to real alveolar bundles. The behavior of one SRSL can be used to estimate the properties of the whole lung, provided it is mechanically representative. ${ }^{38}$ An SRSL must have a sufficient number of AA for accurate estimation of behavior. A $3 \times 3$ SRSL geometry was estimated to be the minimum required to construct a unit cell, based on comparisons of the three SRSLs (Figure 5a). The $3 \times 3$ geometry was the first to exhibit hysteresis ${ }^{39}$ (difference in pressure between the inhalation and the exhalation) due to increased interactions among $\mathrm{AA}$ and increased pathway resistances within the SRSL.

PV behavior was further analyzed to assess the effect of pathways (that connect the AA to the inlet) on the compliance of artificial lungs during a full cycle of respiration. A fully open $3 \times 3$ SRSL and two $3 \times 3$ SRSLs with different pathway geometry (pathway type 1 and pathway type 2 ) were compared to animal lungs (Sprague-Dawley rat and New Zealand white rabbit) during a full cycle of respiration (Figure $5 b)$. Increasing the mean path length of airways within the SRSL was demonstrated to affect the PV behavior. The presence of pathways increases the stiffness of the SRSL. Pathways increase the internal resistance to airflow in an SRSL, as observed in real lungs.

3.3. Modeling Strains in Lung Tissue Using SRSL. To compare the physical deformations of the SRSL with real tissue, full-field strain measurements were performed: DIC was used to map surface deformations of the SRSL, while DVC was performed on rat lung tissue imaged using synchrotron tomography. ${ }^{31}$ A larger SRSL $(5 \times 5$ instead of a $3 \times 3)$ was used to study interactions between multiple AA (minimizing edge effects) for direct comparison to the rat lung data. Major strains of an inflated $5 \times 5$ SRSL were 


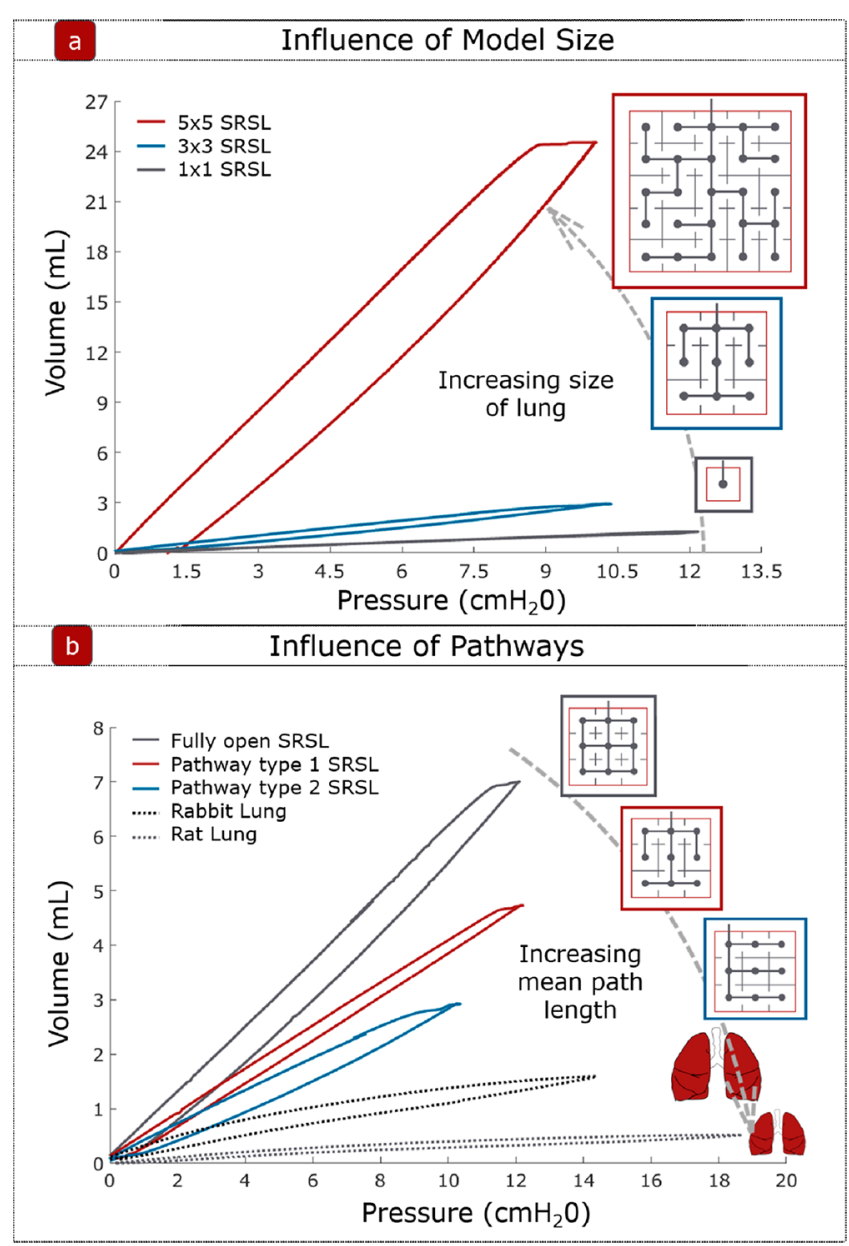

Figure 5. (a) PV curves of different sized SRSL from Figure 2b. (b) PV curves for SRSL with equal volumes that contain different pathways compared to rat and rabbit lung data.

compared to major strains within the lung of a rat (Figure 6a). The alveoli highlighted in the rat lung and the $5 \times 5$ SRSL (black dotted lines, Figure 6a) both exhibited high strains of similar magnitude at the alveolar walls. Although the SRSL comprises a single layer of AA and real lung is formed of threedimensional clusters of alveoli, this SRSL maintains similar mechanical performance to that of a real lung. A single plane of DVC strain data was extracted to enable a direct comparison of a cross-section of lung against the surface DIC measurement of the SRSL.

These results indicate that, despite the SRSL being tested on a different length scale (nearly 3 orders of magnitude larger) than the animal lung, the strain distribution in the SRSL simulates the mammalian lung with strong similarity. Note that this analysis has focused on structural behavior (i.e., solid mechanics) of the SRSL compared to lung tissue, rather than fluid mechanics. Magnitudes of strain (dimensionless) characterize the bulk material behavior at all scales; hence, this characteristic was chosen during this investigation.

3.4. Modeling Respiratory Diseases Using SRSL. When lungs lose their function, due to either constricted and occluded airways or overinflated and overdistended alveoli caused by respiratory diseases, abnormally high strains may arise within local regions of lung tissue. ${ }^{40}$ Different from earlier SRSL geometries produced, the SRSL constructed for studying diseased tissue states consisted of four subunits, each with an individual air inlet to control the degree of inflation (Figure $6 \mathrm{~b})$. In essence, by modulating the degree of inflation in each subunit, the strains produced in diseases such as emphysema (or chronic obstructive pulmonary disease, COPD) can be simulated, ${ }^{41}$ where abnormal strain behavior is observed.

In the first experiment (Figure 6b), all four subunits in the SRSL were inflated with $2 \mathrm{~mL} / \mathrm{AA}$ of dry air and used this DIC frame as the reference frame (or neutral/control state) for the next experiments. Next, Subunit \#1 was held at atmospheric pressure, and Subunits \#2, \#3, and \#4 were inflated with $2 \mathrm{~mL} /$ AA of dry air to simulate an occlusion at Subunit \#1. In this scenario ("Occlusion study" in Figure 6b), the area surrounding the under-inflated subunit (Subunit \#1) exhibited approximately a $10 \%$ increase in major strains. In another experiment ("Overdistension study" in Figure 6b), Subunit \#1 was overinflated with $4 \mathrm{~mL} / \mathrm{AA}$ while inflating Subunits \#2, \#3, and \#4 with $2 \mathrm{~mL} / \mathrm{AA}$ of dry air. The DIC measurements showed that overdistension also produced, as expected, significant bias in the major strains, but this time not only in overinflated subunit, but also the surrounding areas of Subunit $\# 1$. These measurements show that regardless of the level of inflation, uneven pressurization within the SRSL always increases major strains and influences neighboring alveolar strain states. This can lead to serious consequences in the long term after fatigue loading (just from breathing). Similar behavior arises within mammalian lungs (e.g., human lungs) when occlusion/overdistension occurs, leading to critical flaw/ defect propagation. This simple SRSL model can visualize and explore the severity of a given flaw or fault in lung mechanics.

In the overdistension case shown in Figure 6b, major strains across Subunit \#1 were not uniform. To understand the origin of this, the contribution of pathway geometry was investigated by observing the distribution changes in the strain field on the SRSL across each path (Figure $6 \mathrm{c}$ ). All subunits were inflated with $2 \mathrm{~mL} / \mathrm{AA}$ of dry air and overinflated each subunit with a different pathway geometry to $4 \mathrm{~mL} / \mathrm{AA}$ one subunit at a time. For instance, when Subunit \#1 was inflated to $4 \mathrm{~mL} / \mathrm{AA}$, Subunits \#2, \#3, and \#4 were kept at $2 \mathrm{~mL} / \mathrm{AA}$. Common to each overinflated subunit, major strain concentrations occurred in three distinct regions: (i) end of each principle pathway, (ii) areas where pathways split into two or more pathways of equal length, and (iii) AA preceding the AA at the end of the pathway because of pressure build-up. In addition to these three, increased strains in other parts of the SRSL such as the area near the inlet in Subunit \#4 were observed. When investigated closely, deviations in manufacturing that resulted in thinner wall thickness naturally contributed to the concentration of higher stresses. Alveolar wall thickness also varies to a comparable degree $(5-10 \%)$ from one alveolus to the next in nature. ${ }^{39}$ However, the SRSL did achieve uniform strain distribution, independent of manufacturing defects when all subunits were uniformly inflated. From these observations, pathways are deemed critical in determining the points of concentration of higher strains. While manufacture quality can be assessed and, equally, manipulated to identify the role of wall thickness variation (as it commonly changes in disease). The concentration of higher strains in certain regions may be a major contributor to damage initiation in lung tissue, due to a modified local mechanical behavior, caused by a degrading disease or damage sustained after traumatic loading.

3.5. Trauma and Blast. Shock waves produced by a blast may cause severe trauma to lungs. These injuries typically cause tears or tissue rupture of differing size and severity 


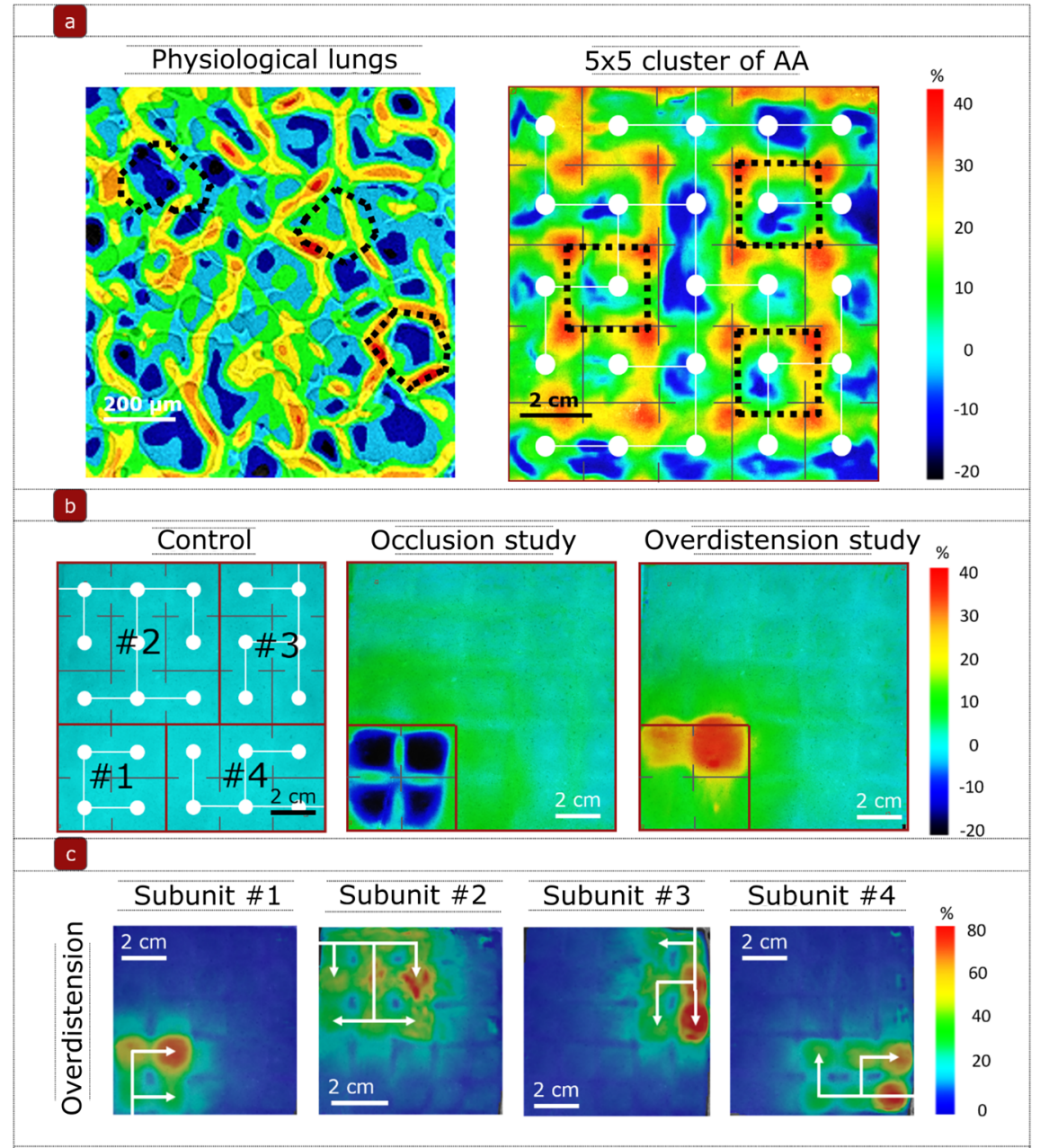

Figure 6. (a) Comparison of the major strains measured in lung tissue and a $5 \times 5$ SRSL from Figure $2 \mathrm{~b}$, acquired by digital volume correlation and digital image correlation, respectively. Example alveoli are highlighted with a dotted black line. Strain fields are overlaid onto the respective raw image; detail of the alveolar structures is visible/highlighted. (b) Three cases of an SRSL with four subunits with a different number of AA. The first case is a control where all alveoli are normally inflated $(2 \mathrm{~mL} / \mathrm{AA})$. The second case describes an under inflation to create a situation of airway occlusion. The third case describes overinflation ( $4 \mathrm{~mL} / \mathrm{AA})$, that is, overdistension. (c) Cases of overdistension are often seen in damaged (trauma) and diseased lungs (COPD).

induced by large dynamic deformations following an explosion. Understanding how shock waves travel through the lung has been a topic of intensive research in recent years. ${ }^{31-33}$ An inflated $3 \times 3$ SRSL, $10 \%$ gelatin hydrogel and rat lungs embedded in $10 \%$ gelatin hydrogel were subjected to a highpressure (120 kPa peak overpressure) shock wave (Figure 7a). The lung sample was embedded in a thin layer of hydrogel to ensure good coupling of the pressure transducer to the lung and to immobilize the lung during the test. The pressure transmitted through each sample was recorded alongside highspeed video footage to capture the dynamic deformation response. See the Supporting Movie for high-speed video footage, at $28000 \mathrm{fps}$, of the response of hydrogel and inflated SRSL subjected to a $120 \mathrm{kPa}$ peak overpressure shock wave. This investigation aimed to identify which material mimics the mammalian lungs with higher accuracy under dynamic loading.

When exposed to the shock wave, the SRSL (inflated with 2 $\mathrm{mL} / \mathrm{AA}$ of dry air) showed more resistance than the hydrogel, which is indicated by its shallower gradient (Figure $7 \mathrm{~b}$ ). The pressures recorded for the hydrogel peaked higher and earlier than both the SRSL and the rat lung tissue. Less compliant than the hydrogel, the SRSL absorbed more energy, slowing down the propagation of the blast wave (potentially sustaining damage). This significant difference makes the SRSL more comparable to mammalian lung tissue than hydrogels. Because of greater interaction of the air-spaces in the SRSL, this offered more comparable resistances observed in the lung experiments. The response of the same $3 \times 3$ SRSL was assessed when subjected to a shock wave of a lower magnitude with $82 \mathrm{kPa}$ peak overpressure (Figure S-3). The SRSL exhibited a response surprisingly similar to the response obtained from a rat lung. The material and geometrical properties of the SRSL must be optimized further to better mimic the response of the mammalian lung at the same blast pressure. However, the results obtained from the blast experiments demonstrate the possibility of using the SRSL to capture basic biomechanical response of animal lung tissue in blast conditions. Further analysis of the SRSL model showed that inflated lungs may be more susceptible to damage due to a blast (Figures S-3 and S4). This is understandable given the lung structure is strain stiffening and so, under inflation, the lung poses greater resistance to deformation. Therefore, the prestrained lung may absorb more energy and sustain greater damage. This 


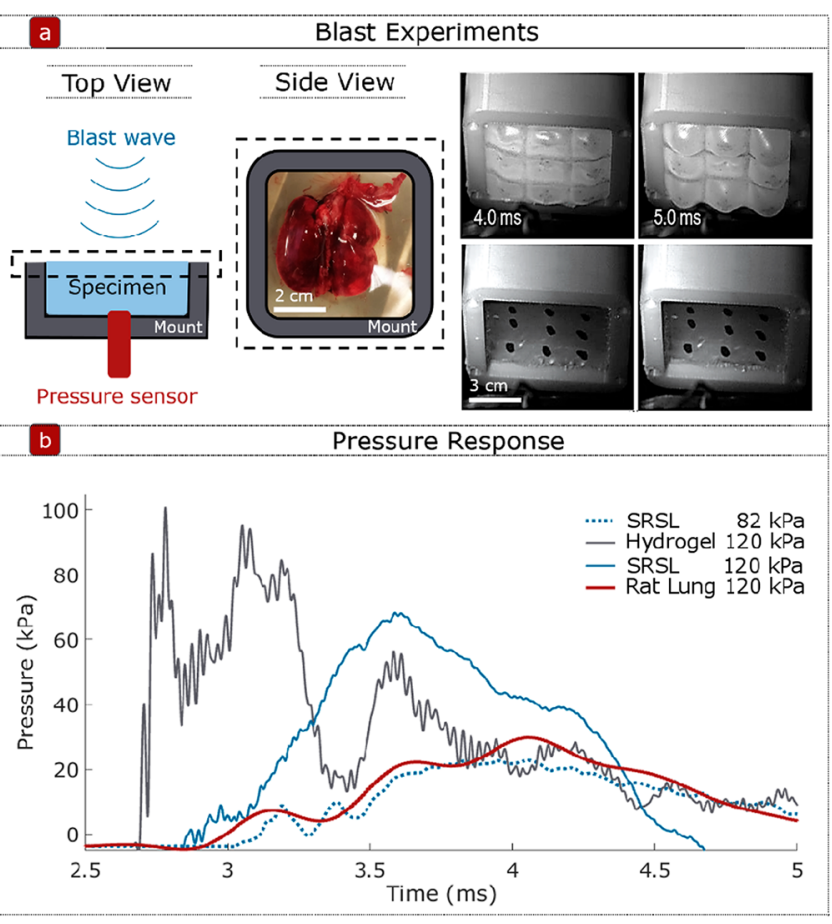

Figure 7. (a) Experimental setup. A specimen such as the lungs of a cadaveric Sprague-Dawley rat $(200-280 \mathrm{~g})$ sit in the mount facing the direction of the blast wave. On the right-hand side, still images were acquired from the high-speed footage to examine the different response of the test samples to shock wave loading. Full display of results at $82 \mathrm{kPa}$ shown in Figure S-3. (b) Pressure transmitted through an inflated SRSL and hydrogel in comparison to rat lungs, when subjected to shock waves to simulate a blast.

information may be valuable for the development of nextgeneration protective technologies and logistics.

\section{CONCLUSION}

The SRSL is an inflatable lung surrogate, simple yet effective for studying lung biomechanics, validated against mammalian lung tissue. It is constructed with highly tunable properties and actuated to simulate breathing action. The SRSL demonstrated a broad range of applications in health, disease, and injury. The SRSL is particularly useful for investigating common lung diseases such as COPD, as the airways can be manipulated to replicate such biomechanical conditions. Equally, the study showed potential to extend toward traumatic/blast injury research.

The fabrication of SRSLs is fast, inexpensive, and simple. The UK-based National Centre for the Replacement, Refinement, and Reduction of Animal in Research (NC3Rs) and other similar institutions aim to develop alternative approaches to research, minimizing the use of animal models. SRSLs offer an animal-free platform for lung biomechanics research. Procedures for licensing and ethical approval are also costly and time-consuming, which can be potentially eliminated by using SRSLs.

The SRSL in its current form has the following three limitations: (i) silicone-based manufacturing using $3 \mathrm{D}$ printed molds yield potential batch-to-batch variability and this increases for producing SRSLs with higher geometrical complexity. (ii) In its current state, the SRSL is actuated by air being pumped into the device rather than drawn in by the motion of expanding lungs. The SRSL is on a ventilator to enable rigid control and direct comparison to PV data collected on excised tissues. In the future, the SRSL will be actuated by other means to initiate the natural motion of the lung to inspire/expire. (iii) This proof-of-concept model SRSL only uses silicones for construction. A more physiologically accurate model, however, would require multiple components synthetic or otherwise to represent the complexity of structural elements in lung tissue. However, strategies and technologies to overcome such barriers are becoming more widely available.

The biofidelity of SRSLs can be improved by introducing microfluidic channels (to carry and distribute liquid media) and house living cells of various types. With these improvements, the SRSL can be transformed into a system similar to emerging organ-a-chip technologies. The silicone elastomers can also be replaced with composite materials or multiple materials to better match the mechanics within the alveolar walls. In the future, the SRSL consisting of living cells in prescribed geometrical structures can be $3 \mathrm{D}$ printed in a single step. With these improvements, the SRSL could allow the study of mass transfer, drug delivery, swelling or tissue rupture, etc. which would, in turn, accelerate the development of therapeutic and preventative interventions for lung disease/ damage by creating a more accessible, realistic testing capability.

\section{ASSOCIATED CONTENT}

\section{S Supporting Information}

The Supporting Information is available free of charge on the ACS Publications website at DOI: 10.1021/acsabm.8b00753.

High-speed video footage from blast trauma experiments (AVI)

Microscopy images of porous silicone, pressure-time histories of blast experiments, schematic of ventilator system and digital image correlation setup, high-speed photography images of inflation conditions (PDF)

\section{AUTHOR INFORMATION}

\section{Corresponding Authors}

*E-mail: f.guder@imperial.ac.uk.

*E-mail: hari.arora@swansea.ac.uk.

\section{ORCID}

\section{Hari Arora: 0000-0002-9790-0907}

\section{Author Contributions}

H.A. and F.G. contributed equally to this work. O.R. manufactured and designed all models and tests, collected the data, composed the results, and wrote the initial manuscript. F.G. and H.A. led the work and are responsible for the initial ideas and their development throughout the entirety of the project. They also both reviewed and finalized the manuscript. H.A. also contributed to the design and procedure of tests.

\section{Notes}

The authors declare no competing financial interest.

\section{ACKNOWLEDGMENTS}

H.A. and F.G. would like to acknowledge the Royal Society for their financial support of this study through their Research Grant Scheme. H.A. would also like to acknowledge the financial support of the Royal British Legion. F.G. and O.R. thank AWE plc (CEMS Kickstart Scheme) for their generous financial support. The authors would like to thank Diamond 
Light Source for beam time at I13-2 under proposal MT12864 and support of LaVision for the reported digital volume correlation lung data. We would also like to thank $\mathrm{M}$. Kasimatis, Prof R.C. Schroter, Dr J.M. Sherwood, and K. Vitharana for their contributions.

\section{REFERENCES}

(1) Weibel, E.; Gomez, D. Architecture of the Human Lung: Use of Quantitative Methods Establishes Fundamental Relations Between Size and Number of Lung Structures. Science 1962, 137, 577-585.

(2) Itoh, H.; Nishino, M.; Hatabu, H. Architecture of the Lung. J. Thorac. Imaging 2004, 19, 221-227.

(3) Sasser, S.; Sattin, R.; Hunt, R.; Krohmer, J. Blast Lung Injury. Prehosp. Emerg. Care 2006, 10, 165-172.

(4) Paré, P.; Mitzner, W. Airway-Parenchymal Interdependence. Compr. Physiol. 2012, 2, 1921-1935.

(5) Dunphy, S.; Bratt, J.; Akram, K.; Forsyth, N.; El Haj, A. Hydrogels for Lung Tissue Engineering: Biomechanical Properties of Thin Collagen-Elastin Constructs. J. Mech. Behav. Biomed. Mater. 2014, 38, 251-259.

(6) Parsa, H.; Karac, A.; Ivankovic, A. A Novel Surrogate Lung Material for Impact Studies: Development and Testing Procedures. J. Biomech. 2017, 63, 74-81.

(7) Wang, X.; Jiang, M.; Zhou, Z.; Gou, J.; Hui, D. 3D Printing of Polymer Matrix Composites: A Review and Prospective. Composites, Part B 2017, 110, 442-458.

(8) Liu, X.; Yuk, H.; Lin, S.; Parada, G.; Zhao, X. Responsive Materials: 3D Printing of Living Responsive Materials and Devices Adv. Adv. Mater. 2018, 30, 1870021.

(9) Hong, S.; Sycks, D.; Chan, H.; Lin, S.; Zhao, X. 3D Printing: 3D Printing of Highly Stretchable and Tough Hydrogels into Complex, Cellularized Structures. Adv. Mater. 2015, 27, 4035-4040.

(10) Huh, D.; Matthews, B.; Mammoto, A.; Montoya-Zavala, M.; Ingber, D. Reconstituting Organ-Level Lung Functions on a Chip. Science 2010, 328, 1662-1668.

(11) Cohrs, N.; Petrou, A.; Loepfe, M.; Yliruka, M.; Stark, W.; et al. A Soft Total Artificial Heart-First Concept Evaluation on a Hybrid Mock Circulation. Artif. Organs 2017, 41, 948-958.

(12) Mac Murray, B.; Futran, C.; Lee, J.; O’Brien, K.; Shepherd, R.; et al. Compliant Buckled Foam Actuators and Application in PatientSpecific Direct Cardiac Compression. Soft Robot. 2018, 5, 99-108.

(13) Roche, E.; Horvath, M.; Wamala, I.; Alazmani, A.; Song, S.; Walsh, C. Soft Robotic Sleeve Supports Heart Function. Sci. Transl. Med. 2017, 9, No. eaaf3925.

(14) Chen, J.; Park, K. Synthesis and Characterization of Superporous Hydrogel Composites. J. Controlled Release 2000, 65, $73-82$.

(15) So, J.; Tayi, A.; Güder, F.; Whitesides, G. Stepped Moduli in Layered Composites. Adv. Funct. Mater. 2014, 24, 7197-7204.

(16) Shepherd, R.; Ilievski, F.; Choi, W.; Morin, S.; Stokes, A.; Whitesides, G. Multigait Soft Robot. Proc. Natl. Acad. Sci. U. S. A. 2011, 108, 20400-20403.

(17) Robinson, S.; Alaie, S.; Sidoti, H.; Auge, J.; Baskaran, L.; Mosadegh, B. Patientspecific Design of a Soft Occluder for the Left Atrial Appendage. Nat. Biomed. Eng. 2018, 2, 8-16.

(18) Polygerinos, P.; Wang, Z.; Galloway, K.; Wood, R.; Walsh, C. Soft robotic Glove for Combined Assistance and At-Home Rehabilitation. Rob. Auton. Syst. 2015, 73, 135-143.

(19) Chen, Q.; Bismarck, A.; Hansen, U.; Junaid, S.; Tran, M.; Harding, S.; Ali, N.; Boccaccini, A. Characterisation of a Soft Elastomer Poly(Glycerol Sebacate) Designed to Match the Mechanical Properties of Myocardial Tissue. Biomaterials 2008, 29, 47-57.

(20) Li, P.; Jiang, S.; Yu, Y.; Yang, J.; Yang, Z. Biomaterial Characteristics and Application of Silicone Rubber and PVA Hydrogels Mimicked in Organ Groups for Prostate Brachytherapy. J. Mech. Behav. Biomed. Mater. 2015, 49, 220-234.
(21) Danelson, K.; Gayzik, F.; Stern, A.; Hoth, J.; Stitzel, J. Design, Development, and Analysis of a Surrogate for Pulmonary Injury Prediction. Ann. Biomed. Eng. 2011, 39, 2560-2567.

(22) Proudfoot, A.; McAuley, D.; Griffiths, M.; Hind, M. Human Models of Acute Lung Injury. Dis. Models \& amp; Mech. 2011, 4, 145153

(23) Andrikakou, P.; Vickraman, K.; Arora, H. On the Behaviour of Lung Tissue under Tension and Compression. Sci. Rep. 2016, 6, 36642.

(24) Hirsch, S.; Posnansky, O.; Papazoglou, S.; Elgeti, T.; Braun, J.; Sack, I. Measurement of Vibration-Induced Volumetric Strain in the Human Lung. Magn. Reson. Med. 2013, 69, 667-674.

(25) Shergold, O.; Fleck, N.; Radford, D. The Uniaxial Stress Versus Strain Response of Pig Skin and Silicone Rubber at Low and High Strain Rates. Int. J. Impact Eng. 2006, 32, 1384-1402.

(26) Böcklin, C.; Baumann, D.; Stuker, F.; Fröhlich, J. Mixing Formula for Tissuemimicking Silicone Phantoms in the Near Infrared. J. Phys. D: Appl. Phys. 2015, 48, 105402.

(27) Peters, A.; Chase, J.; Van Houten, E. Digital Image ElastoTomography: Mechanical Property Estimation of Silicone Phantoms. Med. Biol. Eng. Comput. 2008, 46, 205-212.

(28) Denny, E.; Schroter, R. A Mathematical Model for the Morphology of the Pulmonary Acinus. J. Biomech. Eng. 1996, 118, 210.

(29) Mosadegh, B.; Polygerinos, P.; Keplinger, C.; Wennstedt, S.; Shepherd, R.; Whitesides, G.; et al. Soft Robotics: Pneumatic Networks for Soft Robotics that Actuate Rapidly. Adv. Funct. Mater. 2014, 24, 2109-2109.

(30) Blanch, L. Volume-Pressure Curve of the Respiratory System. Intensive Care Med. 1998, 24, 886-887.

(31) Arora, H.; Nila, A.; Vitharana, K.; Sherwood, J.; Nguyen, T.; Hollis, D.; et al. Microstructural Consequences of Blast Lung Injury Characterized with Digital Volume Correlation. Front. Mater. 2017, 4, 41.

(32) Barnett-Vanes, A.; Sharrock, A.; Eftaxiopoulou, T.; Arora, H.; Macdonald, W.; Rankin, S.; et al. CD43Lo Classical Monocytes Participate in the Cellular Immune Response to Isolated Primary Blast Lung Injury. J. Trauma Acute Care Surg. 2016, 81, 500-511.

(33) Eftaxiopoulou, T.; Barnett-Vanes, A.; Arora, H.; Macdonald, W.; Nguyen, T.; Rankin, S.; et al. Prolonged but not Short-Duration Blast Waves Elicit Acute Inflammation in a Rodent Model of Primary Blast Limb Trauma. Injury 2016, 47, 625-632.

(34) Chen, H.; Kassab, G. Microstructure-Based Constitutive Model of Coronary Artery with Active Smooth Muscle Contraction. Sci. Rep. 2017, 7, 9339.

(35) Mercer, R.; Crapo, J. Spatial Distribution of Collagen and Elastin Fibers in the Lungs. J. Appl. Physiol. 1990, 69, 756-765.

(36) Gibson, L.; Ashby, M. Cellular Solids; Cambridge University Press, 1997.

(37) Khorasani, M. T.; Mirzadeh, H.; Kermani, Z. Wettability of porous polydimethylsiloxane surface: morphology study. Appl. Surf. Sci. 2005, 242, 339-345.

(38) Gusev, A. Representative Volume Element Size for Elastic Composites: A Numerical Study. J. Mech. Phys. Solids 1997, 45, 1449-1459.

(39) Ethier, C.; Simmons, C. Introductory Biomechanics; Cambridge University Press, 2013.

(40) Protti, A.; Cressoni, M.; Santini, A.; Langer, T.; Mietto, C.; Gattinoni, L. Lung Stress and Strain during Mechanical Ventilation. Am. J. Respir. Crit. Care. Med. 2011, 183, 1354-1362.

(41) Ingenito, E.; Tsai, L.; Majumdar, A.; Suki, B. On the Role of Surface Tension in the Pathophysiology of Emphysema. Am. J. Respir. Crit. Care Med. 2005, 171, 300-304. 